Louisiana State University

LSU Digital Commons

$12-1-2019$

\title{
Stabilization and Robustness Analysis for a Chain of Saturating Integrators Arising in the Visual Landing of Aircraft
}

\author{
Laurent Burlion \\ Rutgers University-New Brunswick \\ Michael Malisoff \\ Louisiana State University \\ Frederic Mazenc \\ Laboratoire des Signaux et Systèmes
}

Follow this and additional works at: https://digitalcommons.Isu.edu/mathematics_pubs

\section{Recommended Citation}

Burlion, L., Malisoff, M., \& Mazenc, F. (2019). Stabilization and Robustness Analysis for a Chain of Saturating Integrators Arising in the Visual Landing of Aircraft. Proceedings of the IEEE Conference on Decision and Control, 2019-December, 7013-7018. https://doi.org/10.1109/CDC40024.2019.9029634

This Conference Proceeding is brought to you for free and open access by the Department of Mathematics at LSU Digital Commons. It has been accepted for inclusion in Faculty Publications by an authorized administrator of LSU Digital Commons. For more information, please contact ir@lsu.edu. 
archives-ouvertes

\section{Stabilization and Robustness Analysis for a Chain of Saturating Integrators Arising in the Visual Landing of Aircraft}

Laurent Burlion, Michael Malisoff, Frédéric Mazenc

\section{To cite this version:}

Laurent Burlion, Michael Malisoff, Frédéric Mazenc. Stabilization and Robustness Analysis for a Chain of Saturating Integrators Arising in the Visual Landing of Aircraft. CDC 2019 - 58th IEEE Conference on Decision and Control, Dec 2019, Nice, France. hal-02342711

HAL Id: hal-02342711

https://hal.inria.fr/hal-02342711

Submitted on 1 Nov 2019

HAL is a multi-disciplinary open access archive for the deposit and dissemination of scientific research documents, whether they are published or not. The documents may come from teaching and research institutions in France or abroad, or from public or private research centers.
L'archive ouverte pluridisciplinaire HAL, est destinée au dépôt et à la diffusion de documents scientifiques de niveau recherche, publiés ou non, émanant des établissements d'enseignement et de recherche français ou étrangers, des laboratoires publics ou privés. 


\title{
Stabilization and Robustness Analysis for a Chain of Saturating Integrators Arising in the Visual Landing of Aircraft
}

\author{
Laurent Burlion Michael Malisoff Frédéric Mazenc
}

\begin{abstract}
We study a chain of saturating integrators with imprecise output measurements. Using a recent backstepping approach that leads to pointwise delays in the control and a dynamic extension, we provide an input-to-state stability result using a bounded control of arbitrarily small amplitude. We apply the result to a problem in the visual landing of aircraft.
\end{abstract}

\section{INTRODUCTION}

This paper continues our search for more effective feedback stabilization methods for cases where only imprecise output measurements are available for use in the control. This led to our novel backstepping approach in [14] and [15] where pointwise delays are present in the feedback even if current output values are available, and then our work [11], [12] that uses the preceding backstepping approach to solve a feedback control problem for a chain of saturated integrators with imprecise output measurements using an unbounded control. In the present work, we use our backstepping approach to solve a stabilization problem for a chain of saturating integrators with imprecise measurements using dynamic output feedback controls of arbitrarily small amplitude; see Section II for more on the potential advantages of this work as compared with the method in [11], [12].

The present work is therefore a new development in a long history of research on stabilization under bounded controls. Earlier results on bounded feedback controls include the semi-global state and output feedback stabilization results [23], which involve linear control laws inside saturations. Crucial regional [3] stability results for some linear and nonlinear systems (using LMI methods [2], [22]) were presented in [21]. Other earlier bounded backstepping and forwarding methods lead to globally asymptotically stabilizing controls for some nonlinear systems; see [13] for bounded backstepping, and see [17] and [20] for forwarding methods.

The backstepping designs proposed in [14] and [15] are significantly different from prior backstepping methods, because they circumvent the problem of determining Lie derivatives of the fictitious controls by introducing artificial delays in the control (which are called artificial because

Burlion is with Department of Mechanical and Aerospace Engineering, 98 Brett Road, Rutgers University, Piscataway, NJ 08854, USA. laurent.burlion (at) rutgers.edu

Malisoff is with Department of Mathematics, Louisiana State University, Baton Rouge, LA 70803-4918, USA. malisoff (at) lsu.edu

Mazenc is with Inria EPI DISCO, L2S-CNRS-CentraleSupélec, 3 rue Joliot Curie, 91192, Gif-sur-Yvette, France. frederic.mazenc (at) 12s.centralesupelec.fr

Key words: stabilization, vision-based control, backstepping, aircraft. Burlion was partly supported by ANR VISIOLAND project ANR-13CORD-0012 dedicated to VISIOn based aircraft LANDing techniques. Malisoff was partly supported by NSF Grant 1711299. they are present even if current state values are available for measurement). The artificial delays approach relaxes the smoothness requirement on the fictitious control that was present in previous backstepping approaches.

The advantages of [14] carry over to the present work, which adapts ideas from [14] to a control problem for a chain of saturating integrators for dynamics with outputs that occurs in the vision based [6] landing of aircraft. Since only imprecise measurements of the two first state variables are available in this application, we cannot apply the regional or semi-global results mentioned above, nor [14] or extensions such as [15]. This motivates our new control, which is inspired by the forwarding theory from [16]. Our controls in the present work ensure input-to-state stability with a saturated input. Moreover, for any positive constant $\bar{u}$, we can ensure that our control is bounded by $\bar{u}$.

The notation will be simplified whenever no confusion would arise given the context. Given any constant $T>0, C_{\text {in }}$ denotes the set of all continuous functions $\phi:[-T, 0] \rightarrow \mathbb{R}^{n}$, which we call the set of all initial functions. We define $\Xi_{t} \in C_{\text {in }}$ by $\Xi_{t}(s)=\Xi(t+s)$ for all $\Xi, s \leq 0$, and $t \geq 0$ for which the equality is defined. The Euclidean norm is denoted by $|\cdot|$, and $|\cdot|_{\mathscr{S}}$ (resp., $|\cdot|_{\infty}$ ) denotes the corresponding supremum over any set $\mathscr{S}$ (resp., essential supremum). For each constant $L>0$, we use the usual saturation function $\operatorname{sat}_{L}(x)=\max \{-L, \min \{L, x\}\}$, and $\partial$ will denote a boundary. We also use the standard definitions of input-to-state stability and class $\mathscr{K} \mathscr{L}$ and $\mathscr{K}_{\infty}$ functions, as defined in [7, Chapter 4], and $\mathscr{M}$ denotes the set of all functions of the form $\gamma+c$ where $\gamma \in \mathscr{K}_{\infty}$ and $c \geq 0$ is a constant.

\section{DYNAMICS}

We consider the system

$$
\left\{\begin{array}{l}
\dot{x}_{1}=\operatorname{sat}_{L_{1}}\left(x_{2}\right) \\
\dot{x}_{2}=\operatorname{sat}_{L_{2}}\left(x_{3}\right) \\
\dot{x}_{3}=\operatorname{sat}_{L_{3}}(u),
\end{array}\right.
$$

where $x=\left(x_{1}, x_{2}, x_{3}\right)$ is valued in $\mathbb{R}^{3}$, the input $u$ is valued in $\mathbb{R}$, and $L_{i}>0$ is a constant for $i=1,2,3$, where we use the usual saturation function $\operatorname{sat}_{L}(x)=\max \{-L, \min \{L, x\}\}$ for each constant $L>0$. The outputs are

$$
\begin{aligned}
& y_{1}(t)=\eta(t) x_{1}(t)+\delta_{1}(t) \\
& y_{2}(t)=x_{2}(t)+\delta_{2}(t) \\
& y_{3}(t)=x_{3}(t)
\end{aligned}
$$

where $\delta_{1}, \delta_{2}$ and $\eta$ are unknown but piecewise continuous functions for which there are known constants $\bar{\eta}>1, \bar{\delta}_{1} \geq 0$, 
and $\bar{\delta}_{2} \geq 0$ such that for all $t \geq 0$, we have

$$
\eta(t) \in[1, \bar{\eta}] \text { and }\left|\delta_{i}(t)\right| \leq \bar{\delta}_{i} \text { for } i=1,2
$$

(but see our conclusions section below for remarks in more general cases with sampling or output delays). Requiring $\eta(t) \geq 1$ for all $t \geq 0$ is not restrictive because in practice, $\eta$ will have known positive upper and lower bounds, and then we can divide the formula for the output component $y_{1}$ in (2) by $\inf \{\eta(\ell): \ell \geq 0\}$ so the rescaled $\eta$ and $\delta_{1}$ are such that the rescaled $\eta$ is bounded below by 1 .

Given any constant $\bar{u}>0$, our goal is to construct an output feedback $u$ that is bounded by $\bar{u}$ and that is such that the closed loop system (1) is input-to-state stable with respect to $\delta=\left(\delta_{1}, \delta_{2}\right)$, under the assumption that $\bar{\delta}_{2}<L_{1}$. This contrasts with the objectives in [12], which studied the same system (1) with a sampled version of the same outputs (2), because in [12], the control was not required to be bounded, and in addition, [12] required the more stringent condition $\bar{\delta}_{2}<L_{1}\left(1-e^{-1}\right)^{2} /\left(40\left(1+2 e^{-1}+e^{-2}\right)\right)$ as well as a scaling constant $\lambda>0$ in the control, which are not needed here. Hence, the present paper provides potential advantages over [12], which are made possible by our new dynamical extension in this work which was not present in [12].

Since (1) is not in feedback form, classical backstepping results (e.g., [20]) do not apply. Since $\eta$ is unknown and not necessarily differentiable, bounded backstepping results in [10] and [13] do not apply. Other approaches (e.g., [5], [9], and [16]) do not apply here either. Moreover, one cannot apply [14], which does not allow uncertain measurements. Thus, we believe the problem solved in this work was open and worth solving because of its implications for the visual landing of aircraft that we discuss in Section $\mathrm{V}$ below.

\section{MAIn THEOREM}

Our main result is as follows, in which we can always satisfy our conditions (12) by choosing the positive constants $\bar{\alpha}_{1}, \bar{\beta}_{1}$, and $L_{4}$ small enough and then choosing the $p_{i}$ 's large enough (because of our assumption $\bar{\delta}_{2}<L_{1}$ ), and then for any constant $\bar{u}>0$ we can choose these constants such that the control $u$ is bounded by $L_{4}+\bar{\alpha}_{3}+\bar{\beta}_{2}<\bar{u}$ to satisfy any amplitude restriction on the control $u$ :

Theorem 1: Consider the model (1) with the outputs (2), and assume that $\bar{\delta}_{2}<L_{1}$. Choose the control

$$
\begin{aligned}
& u(t)=\left\{\begin{array}{ll}
0, & t \in[0,2 T) \\
\mathscr{G}\left(t, y_{t}, z_{t}\right), & t \geq 2 T
\end{array}\right. \text { where } \\
& \mathscr{G}\left(t, y_{t}, z_{t}\right)=-\operatorname{sat}_{L_{4}}\left(x_{3}(t)-v_{1}\left(z_{t}\right)-\beta(t)\right) \\
& +v_{2}\left(y_{t}, z_{t}\right)+v_{3}\left(y_{t}, z_{t}\right) \text {, } \\
& v_{1}\left(z_{t}\right)=\frac{k}{\left(1-e^{-k T}\right)^{2}}\left(z_{1}(t)-z_{2}(t)-2 e^{-k T} z_{1}(t-T)\right. \\
& \left.+e^{-k T} z_{2}(t-T)+e^{-2 k T} z_{1}(t-2 T)\right), \\
& v_{2}\left(y_{t}, z_{t}\right)=\frac{k^{2}}{\left(1-e^{-k T}\right)^{2}}\left(-2 z_{1}(t)+z_{2}(t)\right. \\
& +4 e^{-k T} z_{1}(t-T) \\
& -e^{-k T} z_{2}(t-T)-2 e^{-2 k T} z_{1}(t-2 T) \\
& +\phi(t)-2 e^{-k T} \phi(t-T) \\
& \left.+e^{-2 k T} \phi(t-2 T)\right) \text {, and }
\end{aligned}
$$

$$
\begin{aligned}
v_{3}\left(y_{t}, z_{t}\right)= & \frac{k}{1-e^{-k T}}\left(-z_{3}(t)+e^{-k T} z_{3}(t-T)\right. \\
& \left.+\omega(t)-e^{-k T} \omega(t-T)\right)
\end{aligned}
$$

where the $z_{i}$ 's are the states of the dynamic extension

$$
\left\{\begin{array}{l}
\dot{z}_{1}(t)=k\left[-z_{1}(t)+\phi(t)\right] \\
\dot{z}_{2}(t)=k\left[-z_{2}(t)+z_{1}(t)-e^{-k T} z_{1}(t-T)\right] \\
\dot{z}_{3}(t)=k\left[-z_{3}(t)+\omega(t)\right]
\end{array}\right.
$$

with the initial condition $z(\ell)=0$ for all $\ell \in[-2 T, 0]$ and

$$
\begin{aligned}
& \phi(t)=-\frac{\bar{\alpha}_{1}}{p_{1}} \operatorname{sat}_{p_{1}}\left(y_{1}(t)\right) \text { and } \\
& \omega(t)=-\frac{\bar{\beta}_{1}}{p_{2}} \operatorname{sat}_{p_{2}}\left(y_{2}(t)-\alpha(t)\right)
\end{aligned}
$$

and

$\alpha(t)=\frac{z_{2}(t)-e^{-k T} z_{2}(t-T)}{\left(1-e^{-k T}\right)^{2}}$ and $\beta(t)=\frac{z_{3}(t)-e^{-k T} z_{3}(t-T)}{1-e^{-k T}}$

and where the positive constants $p_{i}, T, k, L_{4}, \bar{\alpha}_{1}$, and $\bar{\beta}_{1}$ are chosen such that

$$
\begin{aligned}
& \bar{\beta}_{1}+\bar{\alpha}_{2}<L_{2} \\
& 2 \bar{\alpha}_{1}+\bar{\delta}_{2}<L_{1} \\
& L_{4}+\bar{\alpha}_{3}+\bar{\beta}_{2}<L_{3} \\
& \bar{\eta} T \bar{\alpha}_{1}+\bar{\delta}_{1}<p_{1} \\
& 2 \bar{\alpha}_{1} \bar{\eta}^{2} T<p_{1} \\
& T \bar{\beta}_{1}+\bar{\delta}_{2}<p_{2} \\
& 2 \bar{\beta}_{1} T<p_{2} \\
& \frac{2 T \bar{\alpha}_{1} \bar{\eta}}{p_{1}}\left(\bar{\alpha}_{1}+\xi_{\star}+\frac{\bar{\alpha}_{1}}{p_{1}} \bar{\delta}_{1}\right)+\xi_{\star}+\frac{\bar{\alpha}_{1}}{p_{1}} \bar{\delta}_{1}<\frac{\bar{\alpha}_{1}}{\bar{\eta}}
\end{aligned}
$$

where

$$
\begin{aligned}
& \xi_{\star}=\frac{\bar{\delta}_{2} \bar{\beta}_{1}\left(\bar{\beta}_{1} T+p_{2}\right)}{p_{2}^{2} \sqrt{1-\frac{2}{p_{2}} \bar{\beta}_{1} T}}, \bar{\alpha}_{2}=\frac{2 k \bar{\alpha}_{1}}{1-e^{-k T}}, \\
& \bar{\alpha}_{3}=\frac{4 k^{2} \bar{\alpha}_{1}}{\left(1-e^{-k T}\right)^{2}}, \text { and } \bar{\beta}_{2}=\frac{2 k \bar{\beta}_{1}}{1-e^{-k T}} .
\end{aligned}
$$

Then the control $u$ in (4) is bounded by $L_{4}+\bar{\alpha}_{3}+\bar{\beta}_{2}$, and (1) in closed loop with the control (4) is input-to-state stable with respect to $\delta=\left(\delta_{1}, \delta_{2}\right)$.

\section{Proof of Theorem}

Simple calculations show that the functions $v_{i}$ from (6)-(8) satisfy $v_{1}\left(z_{t}\right)=\dot{\alpha}(t), v_{2}\left(y_{t}, z_{t}\right)=\ddot{\alpha}(t)$, and $v_{3}\left(y_{t}, z_{t}\right)=\dot{\beta}(t)$ for all $t \geq 0$. This allows us to rewrite the control $u$ from (4) as

$$
u(t)=-\operatorname{sat}_{L_{4}}\left(x_{3}(t)-\dot{\alpha}(t)-\beta(t)\right)+\ddot{\alpha}(t)+\dot{\beta}(t)
$$

for all $t \geq 2 T$. Hence, the bound for $u$ follows from the fact that $\alpha$ and $\beta$ in (11) satisfy

$$
\begin{aligned}
& |\alpha(t)| \leq \bar{\alpha}_{1}, \quad|\dot{\alpha}(t)| \leq \bar{\alpha}_{2},|\ddot{\alpha}(t)| \leq \bar{\alpha}_{3}, \\
& |\beta(t)| \leq \bar{\beta}_{1}, \text { and }|\dot{\beta}(t)| \leq \bar{\beta}_{2}
\end{aligned}
$$

for all $t \geq 2 T$. Our proof of the formulas (15) (in Appendix 1 below) use the fact that we can apply variation of parameters (to the system $\dot{q}=k(-q+b)$ with the choice $(q(t), b(t))=\left(z_{3}(t), \omega(t)\right)$, then with the choice $(q(t), b(t))=$ 
$\left(z_{2}(t), z_{1}(t)-e^{-k T} z_{1}(t-T)\right)$, and finally with the choice $\left.(q(t), b(t))=\left(z_{1}(t), \phi(t)\right)\right)$ to prove that

$$
\begin{aligned}
& \alpha(t)=\frac{k^{2}}{\left(1-e^{-k T}\right)^{2}} \int_{t-T}^{t} \int_{m-T}^{m} e^{k(\ell-t)} \phi(\ell) \mathrm{d} \ell \mathrm{d} m \text { and } \\
& \beta(t)=\frac{k}{1-e^{-k T}} \int_{t-T}^{t} e^{k(\ell-t)} \omega(\ell) \mathrm{d} \ell
\end{aligned}
$$

for all $t \geq 2 T$. We also use the formulas (16) in the rest of the proof as well.

The remainder of the proof is organized as follows. In the first step, we use a change of variables that produces a useful cascaded system with a globally asymptotically stable subsystem. In the second step, we perform a Lyapunov function analysis using states of this new system. In the third step, we use results from the first two steps to find useful bounds on the states of the original system. In the final step, we use linear growth properties of the closed loop system to transform the preceding estimates into the required input-tostate stability estimate.

First step. We use the new variables

$$
\begin{aligned}
& \xi_{2}(t)=x_{2}(t)-\alpha(t) \\
& \xi_{3}(t)=x_{3}(t)-\dot{\alpha}(t)-\beta(t) .
\end{aligned}
$$

Direct calculations give

$$
\left\{\begin{array}{l}
\dot{x}_{1}(t)=\operatorname{sat}_{L_{1}}\left(\alpha(t)+\xi_{2}(t)\right) \\
\dot{\xi}_{2}(t)=\operatorname{sat}_{L_{2}}\left(\beta(t)+\xi_{3}(t)+\dot{\alpha}(t)\right)-\dot{\alpha}(t) \\
\dot{\xi}_{3}(t)=\operatorname{sat}_{L_{3}}(u)-\ddot{\alpha}(t)-\dot{\beta}(t) .
\end{array}\right.
$$

Notice that the system (18) in closed-loop with (4) is forward complete, by the forward completeness of our dynamic extension (9) (which follows from the boundedness of the nonlinear terms in (9)). Also, (15) and (12c) imply that $|u(t)|=\left|-\operatorname{sat}_{L_{4}}\left(\xi_{3}(t)\right)+\ddot{\alpha}(t)+\dot{\beta}(t)\right| \leq L_{4}+\bar{\alpha}_{3}+\bar{\beta}_{2}<L_{3}$ holds for all $t \geq 2 T$. As an immediate consequence, the system (18) in closed loop with the feedback defined in (4) admits the representation

$$
\left\{\begin{array}{l}
\dot{x}_{1}(t)=\operatorname{sat}_{L_{1}}\left(\alpha(t)+\xi_{2}(t)\right) \\
\dot{\xi}_{2}(t)=\operatorname{sat}_{L_{2}}\left(\beta(t)+\xi_{3}(t)+\dot{\alpha}(t)\right)-\dot{\alpha}(t) \\
\dot{\xi}_{3}(t)=-\operatorname{sat}_{L_{4}}\left(\xi_{3}(t)\right)
\end{array}\right.
$$

for all $t \geq 2 T$. Thus $\xi_{3}(t)$ converges asymptotically to 0 . We deduce from (12a) and the bounds (15) that there is a class $\mathscr{M}$ function $T_{b}:[0,+\infty) \rightarrow[2 T,+\infty)$ (depending on $\bar{\beta}_{1}$ and $\left.\bar{\alpha}_{2}\right)$ such that for all $t \geq T_{b}(|\xi(0)|)$, we have $\mid \beta(t)+\xi_{3}(t)+$ $\dot{\alpha}(t)\left|\leq \bar{\beta}_{1}+\bar{\alpha}_{2}+\right| \xi_{3}(t) \mid<L_{2}$. Hence,

$$
\left\{\begin{array}{l}
\dot{x}_{1}(t)=\operatorname{sat}_{L_{1}}\left(\alpha(t)+\xi_{2}(t)\right) \\
\dot{\xi}_{2}(t)=\beta(t)+\xi_{3}(t)
\end{array}\right.
$$

for all $t \geq T_{b}(|\xi(0)|)$.

Using the formulas $y_{1}(t)=\eta(t) x_{1}(t)+\delta_{1}(t)$ and $y_{2}(t)-$ $\alpha(t)=x_{2}(t)+\delta_{2}(t)-\alpha(t)=\xi_{2}(t)+\delta_{2}(t)$ for our output components from (2) (which follow from (17)), and also using our formulas for $\phi$ and $\omega$ from (10) and (16), we can then rewrite (20) as

$$
\begin{aligned}
& \dot{x}_{1}(t)= \\
& \operatorname{sat}_{L_{1}}\left(-\frac{k^{2} \bar{\alpha}_{1}}{\left(1-e^{-k T}\right)^{2} p_{1}} \int_{t-T}^{t} \int_{m-T}^{m} e^{k(\ell-t)} \operatorname{sat}_{p_{1}}\left(\eta(\ell) x_{1}(\ell)\right.\right. \\
& \left.\left.+\delta_{1}(\ell)\right) \mathrm{d} \ell \mathrm{d} m+\xi_{2}(t)\right)
\end{aligned}
$$

and

$$
\begin{aligned}
\dot{\xi}_{2}(t)= & -\frac{\bar{\beta}_{1} k}{p_{2}\left(1-e^{-k T}\right)} \int_{t-T}^{t} e^{k(\ell-t)} \operatorname{sat}_{p_{2}}\left(\xi_{2}(\ell)\right. \\
& \left.+\delta_{2}(\ell)\right) \mathrm{d} \ell+\xi_{3}(t)
\end{aligned}
$$

for all $t \geq T_{b}(|\xi(0)|)$.

We also rewrite the $\xi_{2}$-subsystem as

$$
\begin{aligned}
& \dot{\xi}_{2}(t)= \\
& -\frac{\bar{\beta}_{1} k}{p_{2}\left(1-e^{-k T}\right)} \int_{t-T}^{t} e^{k(\ell-t)} \operatorname{sat}_{p_{2}}\left(\xi_{2}(\ell)\right) \mathrm{d} \ell+\mathscr{J}_{2}(t),
\end{aligned}
$$

where

$$
\begin{aligned}
& \mathscr{J}_{2}(t)=\frac{\bar{\beta}_{1} k}{p_{2}\left(1-e^{-k T}\right)} \int_{t-T}^{t} e^{k(\ell-t)}\left[\operatorname{sat}_{p_{2}}\left(\xi_{2}(\ell)\right)\right. \\
& \left.-\operatorname{sat}_{p_{2}}\left(\xi_{2}(\ell)+\delta_{2}(\ell)\right)\right] \mathrm{d} \ell+\xi_{3}(t) .
\end{aligned}
$$

We rewrite the $x_{1}$ subsystem as

$$
\begin{aligned}
& \dot{x}_{1}(t)=\mathscr{J}_{1}(t)+ \\
& \operatorname{sat}_{L_{1}}\left(-\frac{k^{2} \bar{\alpha}_{1}}{\left(1-e^{-k T}\right)^{2} p_{1}} \int_{t-T}^{t} \int_{m-T}^{m} e^{k(\ell-t)} P_{1}(\ell) \mathrm{d} \ell \mathrm{d} m\right),
\end{aligned}
$$

where $P_{1}(\ell)=\operatorname{sat}_{p_{1}}\left(\eta(\ell) x_{1}(\ell)\right)$ and

$\mathscr{J}_{1}(t)=$

$\operatorname{sat}_{L_{1}}\left(-\frac{k^{2} \bar{\alpha}_{1}}{\left(1-e^{-k T}\right)^{2} p_{1}} \int_{t-T}^{t} \int_{m-T}^{m} e^{k(\ell-t)} \operatorname{sat}_{p_{1}}\left(\eta(\ell) x_{1}(\ell)\right.\right.$

$\left.\left.+\delta_{1}(\ell)\right) \mathrm{d} \ell \mathrm{d} m+\xi_{2}(t)\right)$

$-\operatorname{sat}_{L_{1}}\left(-\frac{k^{2} \bar{\alpha}_{1}}{\left(1-e^{-k T}\right)^{2} p_{1}} \int_{t-T}^{t} \int_{m-T}^{m} e^{k(\ell-t)} P_{1}(\ell) \mathrm{d} \ell \mathrm{d} m\right)$.

By (12b), we have $\bar{\alpha}_{1}<L_{1}$, and $\int_{t-T}^{t} \int_{m-T}^{m} e^{k(\ell-t)} \mathrm{d} \ell \mathrm{d} m=$ $\frac{1}{k^{2}}\left(1-e^{-k T}\right)^{2}$ holds for all $t \geq 0$ and sat $p_{1}$ is bounded by $p_{1}$, so (25) gives

$\dot{x}_{1}(t)=\mathscr{J}_{1}(t)$

$-\frac{k^{2} \bar{\alpha}_{1}}{\left(1-e^{-k T}\right)^{2} p_{1}} \int_{t-T}^{t} \int_{m-T}^{m} e^{k(\ell-t)} \operatorname{sat}_{p_{1}}\left(\eta(\ell) x_{1}(\ell)\right) \mathrm{d} \ell \mathrm{d} m$

for all $t \geq T_{b}(|\xi(0)|)$. Finally, since saturations have the global Lipschitz constant 1 , we can check that

$$
\begin{aligned}
& \left|\mathscr{J}_{2}(t)\right| \leq\left|\xi_{3}(t)\right|+\frac{\bar{\beta}_{1}}{p_{2}} \overline{\boldsymbol{\delta}}_{2} \text { and } \\
& \left|\mathscr{J}_{1}(t)\right| \leq\left|\xi_{2}(t)\right|+\frac{\bar{\alpha}_{1}}{p_{1}} \bar{\delta}_{1} \text { for all } t \geq T_{b}(|\xi(0)|),
\end{aligned}
$$

by the formula $\int_{t-T}^{t} e^{k(\ell-t)} \mathrm{d} \ell=\frac{1}{k}\left(1-e^{-k T}\right)$. We next analyze the stability properties of the system (23).

Using (23), it follows that, for all $t \geq T_{b}(|\xi(0)|)+T$,

$$
\begin{aligned}
\dot{\xi}_{2}(t)= & -\frac{\bar{\beta}_{1}}{p_{2}} \operatorname{sat}_{p_{2}}\left(\xi_{2}(t)\right)+\mathscr{R}_{1}(t)+\mathscr{J}_{2}(t), \text { where } \\
& \mathscr{R}_{1}(t)=\frac{\bar{\beta}_{1} k}{p_{2}\left(1-e^{-k T}\right)} \int_{t-T}^{t} e^{k(\ell-t)}\left[\operatorname{sat}_{p_{2}}\left(\xi_{2}(t)\right)\right. \\
& \left.-\operatorname{sat}_{p_{2}}\left(\xi_{2}(t)-\int_{\ell}^{t} \dot{\xi}_{2}(s) \mathrm{d} s\right)\right] \mathrm{d} \ell .
\end{aligned}
$$

From (22), we deduce that $\left|\dot{\xi}_{2}(t)\right| \leq\left|\xi_{3}(t)\right|+\bar{\beta}_{1}$. As a consequence, for all $t \geq T_{b}(|\xi(0)|)+T$, we have

$$
\begin{aligned}
& \left|\mathscr{R}_{1}(t)\right| \\
& \leq \frac{\bar{\beta}_{1} k}{p_{2}\left(1-e^{-k T}\right)} \int_{t-T}^{t} e^{k(\ell-t)}(t-\ell) \mathrm{d} \ell\left(\left|\xi_{3}\right|_{[t-T, t]}+\bar{\beta}_{1}\right) \\
& \leq \frac{\bar{\beta}_{1} T}{p_{2}}\left(\left|\xi_{3}\right|_{[t-T, t]}+\bar{\beta}_{1}\right) .
\end{aligned}
$$


This inequality and the first inequality in (28) yield

$$
\begin{aligned}
& \left|\mathscr{R}_{1}(t)\right|+\left|\mathscr{J}_{2}(t)\right| \leq \\
& \frac{\bar{\beta}_{1}}{p_{2}}\left[T\left(\left|\xi_{3}\right|_{[t-T, t]}+\bar{\beta}_{1}\right)+\bar{\delta}_{2}\right]+\left|\xi_{3}(t)\right|
\end{aligned}
$$

for all $t \geq T_{b}(|\xi(0)|)+T$. Since the $\xi_{3}$ subsystem is globally asymptotically stable to 0 , we deduce from (12f) that there is a class $\mathscr{M}$ function $T_{c}:[0,+\infty) \rightarrow[0,+\infty)$ and a constant $\delta_{0}>0$ such that

$$
\begin{aligned}
& \sup _{\ell \geq T_{C}(|\xi(0)|)}\left(\left|\mathscr{R}_{1}(\ell)\right|+|\mathscr{J}(\ell)|\right) \\
& \leq \frac{\bar{\beta}_{1}}{p_{2}}\left[T \bar{\beta}_{1}+\bar{\delta}_{2}\right]+\delta_{0}<\bar{\beta}_{1} .
\end{aligned}
$$

Hence, by (29a), there is a class $\mathscr{M}$ function $T_{d}:[0,+\infty) \rightarrow$ $[0,+\infty)$ such that $T_{d}(s) \geq T_{b}(s)+T$ for all $s \geq 0$ and such that $\left|\xi_{2}(t)\right|<p_{2}$ for all $t \geq T_{d}(|\xi(0)|)$, because $\xi_{2}>0$ (resp., $\left.<0\right)$ when $\xi_{2}(t) \leq-p_{2}$ (resp., $\geq p_{2}$ ). Then for all $t \geq T_{d}(|\xi(0)|)$, we have sat $p_{2}\left(\xi_{3}(t)\right)=\xi_{3}(t)$ and therefore also

$$
\dot{\xi}_{2}(t)=-\frac{\bar{\beta}_{1}}{p_{2}} \xi_{2}(t)+\mathscr{R}_{1}(t)+\mathscr{J}_{2}(t) .
$$

Second step. This step will use the candidate Lyapunov function $\Upsilon\left(\xi_{2}\right)=\frac{1}{2} \xi_{2}^{2}$. By (29b), we get

$$
\begin{aligned}
\left|\mathscr{R}_{1}(t)\right| & \leq \frac{\bar{\beta}_{1} k}{p_{2}\left(1-e^{-k T}\right)} \int_{t-T}^{t} e^{k(\ell-t)} \int_{\ell}^{t}\left|\dot{\xi}_{2}(s)\right| \mathrm{d} s \mathrm{~d} \ell \\
& \leq \frac{\bar{\beta}_{1}}{p_{2}} \int_{t-T}^{t}\left|\dot{\xi}_{2}(s)\right| \mathrm{d} s
\end{aligned}
$$

for all $t \geq T_{d}(|\xi(0)|)$. By using (23) to upper bound $\left|\dot{\xi}_{2}(s)\right|$ in (34), we deduce from the second inequality in (34) that for all $t \geq T_{d}(|\xi(0)|)$, we have

$$
\begin{aligned}
\left|\mathscr{R}_{1}(t)\right| \leq & \frac{\bar{\beta}_{1}^{2} k}{p_{2}^{2}\left(1-e^{-k T}\right)} \int_{t-T}^{t} \int_{s-T}^{s} e^{k(\ell-s)} \mathrm{d} \ell \mathrm{d} s \\
& \times \sup _{m \in[t-2 T, t]}\left|\xi_{2}(m)\right|+\frac{\bar{\beta}_{1}}{p_{2}} \int_{t-T}^{t}\left|\mathscr{J}_{2}(s)\right| \mathrm{d} s \\
\leq & \frac{\bar{\beta}_{1}^{2} T}{p_{2}^{2}} \sup _{m \in[t-2 T, t]}\left|\xi_{2}(m)\right| \\
& +\frac{\bar{\beta}_{1} T}{p_{2}}\left(\frac{\bar{\beta}_{1}}{p_{2}} \bar{\delta}_{2}+\left|\xi_{3}\right|_{[t-T, t]}\right),
\end{aligned}
$$

where the last inequality is a consequence of the first inequality in (28). By using the first inequality in (28) and (35) to bound $\mathscr{R}_{1}$ and $\mathscr{J}_{2}$ from (33), we easily deduce that for any constant $\omega_{0}>0$, we have

$$
\begin{aligned}
\dot{\Upsilon}(t) \leq & \frac{\bar{\beta}_{1}}{p_{2}}\left(-\xi_{2}^{2}(t)+\frac{\bar{\beta}_{1} T}{p_{2}} \sup _{m \in[t-2 T, t]} \xi_{2}^{2}(m)\right. \\
& \left.+\left|\xi_{2}(t)\right|\left[\left(\frac{\bar{\beta}_{1} T}{p_{2}}+1\right) \frac{\bar{\beta}_{1}}{p_{2}} \overline{\boldsymbol{\delta}}_{2}+\mu(|\xi(0)|, t)\right]\right) \\
\leq & \frac{\bar{\beta}_{1}}{p_{2}}\left(-\Upsilon\left(\xi_{2}(t)\right)+\frac{2 \bar{\beta}_{1} T}{p_{2}} \sup _{m \in[t-2 T, t]} \Upsilon\left(\xi_{2}(m)\right)\right. \\
& \left.+\frac{B_{*}(t)}{2}\right),
\end{aligned}
$$

along all solutions of (33) for all $t \geq T_{d}(\mid \xi(0 \mid)+T$, where

$$
\begin{aligned}
B_{*}(t)= & \left(1+\omega_{0}\right)\left(\frac{\bar{\beta}_{1} T}{p_{2}}+1\right)^{2} \frac{\bar{\beta}_{1}^{2}}{p_{2}^{2}} \bar{\delta}_{2}^{2} \\
& +\left[\left(1+\frac{1}{\omega_{0}}\right) \mu^{2}(|\xi(0)|, t)\right],
\end{aligned}
$$

and where the last inequality in (36) used the triangle inequality $a b \leq \frac{1}{2} a^{2}+\frac{1}{2} b^{2}$ with $a=\left|\xi_{2}(t)\right|$ and $b$ being the quantity in squared brackets in (36) (followed by a use of the relation $(r+s)^{2} \leq\left(1+\omega_{0}\right) r^{2}+\left(1+\left(1 / \omega_{0}\right)\right) s^{2}$ for suitable $r$ and $s$, and where $\mu$ is a class $\mathscr{K} \mathscr{L}$ function. Since $\mu \in \mathscr{K} \mathscr{L}$, we can then find a class $\mathscr{M}$ function $T_{d}^{\sharp}:[0,+\infty) \rightarrow[0,+\infty)$ such that $T_{d}^{\sharp}(s) \geq T_{d}(s)$ for all $s \geq 0$ and such that $\mu(|\xi(0)|, t) \leq \omega_{0}^{2}$ for all $t \geq T_{d}^{\sharp}(|\xi(0)|)$, and therefore such that the quantity in squared brackets in (37) is bounded above by $\omega_{0}+\omega_{0}^{2}$ for all $t \geq T_{d}^{\sharp}(|\xi(0)|)$. Therefore, for any $\varepsilon>0$, we can choose $\omega_{0}>0$ small enough such that

$$
\sqrt{B_{*}(t) \bar{\beta}_{1} /\left(p_{2}\left(c_{1}-c_{2}\right)\right)}<\xi_{\star}(1+\varepsilon)
$$

for all $t \geq T_{d}^{\sharp}(|\xi(0)|)$, where $\xi_{\star}$ was defined in (13), $c_{1}=\bar{\beta}_{1} / p_{2}$, and $c_{2}=2 \bar{\beta}_{1}^{2} T / p_{2}^{2}$. Since $(12 \mathrm{~g})$ ensures that $1>2 \bar{\beta}_{1} T / p_{2}$, it follows from Lemma A.1 in Appendix 2 (applied to $v(t)=\Upsilon\left(\xi_{2}\left(t+T_{d}^{\sharp}(|\xi(0)|)+T\right)\right)$, and with the preceding choices of $c_{1}$ and $c_{2}$ and the choice $\Delta_{1}=$ $\frac{1}{2}\left(\bar{\beta}_{1} / p_{2}\right) \sup \left\{B_{*}(t): t \geq T_{d}^{\sharp}(|\xi(0)|\}\right)$ that

$$
\left|\xi_{2}(t)\right| \leq \xi_{\star}(1+\varepsilon)+\mu_{0}\left(|x(0)|+|\delta|_{\infty}, t\right)
$$

holds for all $t \geq T_{d}^{\sharp}(|\xi(0)|)$; this is done by using the formulas (16)-(17) to find a function $\gamma \in \mathscr{K}_{\infty}$ such that $|\xi(0)| \leq$ $\gamma\left(|x(0)|+|\delta|_{\infty}\right)$ for all initial states, in order to find $\mu_{0}$.

The next part of the proof is devoted to the $x_{1}$-subsystem (27). First, we deduce from (27) that $\left|\dot{x}_{1}(t)\right| \leq \bar{\alpha}_{1}+\left|\mathscr{J}_{1}(t)\right| \leq$ $\bar{\alpha}_{1}+\left|\xi_{2}(t)\right|+\frac{\bar{\alpha}_{1}}{p_{1}} \bar{\delta}_{1}$, using the second inequality in (28), so (39) gives

$$
\left|\dot{x}_{1}(t)\right| \leq \bar{\alpha}_{1}+\xi_{\star}(1+\varepsilon)+\frac{\bar{\alpha}_{1}}{p_{1}} \overline{\boldsymbol{\delta}}_{1}+\mu_{0}\left(|x(0)|+|\delta|_{\infty}, t\right)
$$

for all $t \geq T_{d}^{\sharp}(|\xi(0)|)$. From (27), it follows that for all $t \geq$ $T_{d}^{\sharp}(|\xi(0)|)$, we have

$$
\begin{aligned}
& \dot{x}_{1}(t)=\mathscr{R}_{2}(t)+\mathscr{J}_{1}(t) \\
& -\frac{k^{2} \bar{\alpha}_{1}}{\left(1-e^{-k T}\right)^{2} p_{1}} \int_{t-T}^{t} \int_{m-T}^{m} e^{k(\ell-t)} \operatorname{sat}_{p_{1}}\left(\eta(\ell) x_{1}(t)\right) \mathrm{d} \ell \mathrm{d} m, \\
& \quad \text { where } \mathscr{R}_{2}(t)= \\
& \quad-\frac{k^{2} \bar{\alpha}_{1}}{\left(1-e^{-k T}\right)^{2} p_{1}} \int_{t-T}^{t} \int_{m-T}^{m} e^{k(\ell-t)}\left[\operatorname{sat}_{p_{1}}\left(\eta(\ell) x_{1}(\ell)\right)\right. \\
& \left.\quad-\operatorname{sat}_{p_{1}}\left(\eta(\ell) x_{1}(t)\right)\right] \mathrm{d} \ell \mathrm{d} m .
\end{aligned}
$$

As an immediate consequence, for all $t \geq T_{d}^{\sharp}(|\xi(0)|)$, we have

$$
\begin{aligned}
& \left|\mathscr{R}_{2}(t)\right| \leq \\
& \frac{k^{2} \bar{\alpha}_{1}}{\left(1-e^{-k T}\right)^{2} p_{1}} \bar{\eta} \int_{t-T}^{t} \int_{m-T}^{m} e^{k(\ell-t)}\left|x_{1}(t)-x_{1}(\ell)\right| \mathrm{d} \ell \mathrm{d} m \\
& \leq \frac{\bar{\alpha}_{1} \bar{\eta}}{p_{1}} \int_{t-2 T}^{t}\left|\dot{x}_{1}(s)\right| \mathrm{d} s,
\end{aligned}
$$

since the Fundamental Theorem of Calculus gives $\mid x_{1}(t)-$ $x_{1}(\ell)\left|\leq \int_{t-2 T}^{t}\right| \dot{x}_{1}(s) \mid \mathrm{d} s$ for all $\ell \in[t-2 T, t]$.

From (40), it follows that for all $t \geq T_{d}^{\sharp}(|\xi(0)|)+2 T$, we have

$$
\begin{aligned}
& \left|\mathscr{R}_{2}(t)\right| \leq \frac{2 T \bar{\alpha}_{1} \bar{\eta}}{p_{1}}\left(\bar{\alpha}_{1}+\xi_{\star}(1+\varepsilon)+\mu_{0}^{b}\left(|x(0)|+|\delta|_{\infty}, t\right)\right. \\
& \left.+\frac{\bar{\alpha}_{1}}{p_{1}} \bar{\delta}_{1}\right)
\end{aligned}
$$


where $\mu_{0}^{b}(s, t)=\mu_{0}(s, t-2 T)$. Combining (43) and the second inequality in (28) with (39), we obtain a class $\mathscr{M}$ function $T_{d}^{\sharp \sharp}:[0,+\infty) \rightarrow[0,+\infty)$ such that

$$
\sup _{t \geq T_{d}^{\sharp \sharp}(|\xi(0)|)}\left(\left|\mathscr{R}_{2}(t)\right|+\left|\mathscr{J}_{1}(t)\right|\right)<\frac{\bar{\alpha}_{1}}{\bar{\eta}},
$$

where the last inequality is a consequence of (12h) and by choosing $\varepsilon \in(0,1)$ to be small enough. Next observe that when $x_{1}(t) \geq p_{1} / \bar{\eta}$, then, bearing in mind that $\eta$ is lower bounded by 1 , we deduce from (41a) that

$$
\begin{aligned}
\dot{x}_{1}(t) \leq & -\frac{k^{2} \bar{\alpha}_{1}}{\left(1-e^{-k T}\right)^{2} p_{1}} \int_{t-T}^{t} \int_{m-T}^{m} e^{k(\ell-t)} \frac{p_{1}}{\bar{\eta}} \mathrm{d} \ell \mathrm{d} m \\
& +\mathscr{R}_{2}(t)+\mathscr{J}_{1}(t) \\
= & -\frac{\bar{\alpha}_{1}}{\bar{\eta}}+\mathscr{R}_{2}(t)+\mathscr{J}_{1}(t)<0
\end{aligned}
$$

for all $t \geq T_{d}^{\sharp \sharp}(|\xi(0)|)$, where the second inequality is a consequence of (44). Similarly, one can prove that when $x_{1}(t) \leq-p_{1} / \bar{\eta}$ then $\dot{x}_{1}(t)>0$. Hence, there is class $\mathscr{M}$ function $T_{e}:[0,+\infty) \rightarrow[0,+\infty)$ such that $\left|\eta(\ell) x_{1}(t)\right|<p_{1}$ for all $t \geq T_{e}(|\xi(0)|)$ and $\ell \geq 0$. It follows from (41a) that

$$
\begin{aligned}
\dot{x}_{1}(t)= & -\frac{k^{2} \bar{\alpha}_{1}}{\left(1-e^{-k T}\right)^{2} p_{1}} \int_{t-T}^{t} \int_{m-T}^{m} e^{k(\ell-t)} \eta(\ell) x_{1}(t) \mathrm{d} \ell \mathrm{d} m \\
& +\mathscr{R}_{2}(t)+\mathscr{J}_{1}(t)
\end{aligned}
$$

for all $t \geq T_{e}(|\xi(0)|)$. We can assume that $T_{e}(s) \geq T_{d}^{\sharp}(s)+2 T$ for all $s \geq 0$.

Let us consider the candidate Lyapunov function $v\left(x_{1}\right)=$ $\frac{1}{2} x_{1}^{2}$. Since $\eta$ is lower bounded by 1 , we deduce from the second inequality in (28) and (42) that

$$
\begin{aligned}
\dot{v}(t) \leq & -\frac{\bar{\alpha}_{1}}{p_{1}} x_{1}^{2}(t)+x_{1}(t)\left[\mathscr{R}_{2}(t)+\mathscr{J}_{1}(t)\right] \\
\leq & -\frac{\bar{\alpha}_{1}}{p_{1}} x_{1}^{2}(t)+\left|x_{1}(t)\right| \frac{\bar{\alpha}_{1} \bar{\eta}}{p_{1}} \int_{t-2 T}^{t}\left|\dot{x}_{1}(s)\right| \mathrm{d} s \\
& +\left|x_{1}(t)\right|\left(\left|\xi_{2}(t)\right|+\frac{\bar{\alpha}_{1}}{p_{1}} \bar{\delta}_{1}\right)
\end{aligned}
$$

holds along all solutions of (46) for all $t \geq T_{e}(|\xi(0)|)$. From (27), we deduce that

$$
\begin{aligned}
\left|\dot{x}_{1}(t)\right| \leq & \frac{k^{2} \bar{\alpha}_{1}}{\left(1-e^{-k T}\right)^{2} p_{1}} \int_{t-T}^{t} \int_{m-T}^{m} e^{k(\ell-t)} \bar{\eta}\left|x_{1}(\ell)\right| \mathrm{d} \ell \mathrm{d} m \\
& +\left|\mathscr{J}_{1}(t)\right| \\
\leq & \frac{\bar{\alpha}_{1} \bar{\eta}}{p_{1}} \sup _{m \in[t-2 T, t]}\left|x_{1}(m)\right|+\left|\xi_{2}(t)\right|+\frac{\bar{\alpha}_{1}}{p_{1}} \bar{\delta}_{1}
\end{aligned}
$$

for all $t \geq T_{e}(|\xi(0)|)$, by our bound $\bar{\eta}$ on $\eta$, and where the second inequality is by (28), so (47) and the choice

$$
\mathscr{H}(s)=\frac{\bar{\alpha}_{1} \bar{\eta}}{p_{1}} \sup _{m \in[s-2 T, s]}\left|x_{1}(m)\right|+\left|\xi_{2}(s)\right|
$$

give

$$
\begin{aligned}
\dot{v}(t) \leq & -\frac{\bar{\alpha}_{1}}{p_{1}} x_{1}^{2}(t)+\left|x_{1}(t)\right| \frac{\bar{\alpha}_{1} \bar{\eta}}{p_{1}} \int_{t-2 T}^{t} \mathscr{H}(s) \mathrm{d} s \\
& +\left|x_{1}(t)\right|\left(\frac{2 T \bar{\alpha}_{1} \bar{\eta}}{p_{1}} \frac{\bar{\alpha}_{1}}{p_{1}} \bar{\delta}_{1}+\left|\xi_{2}(t)\right|+\frac{\bar{\alpha}_{1}}{p_{1}} \bar{\delta}_{1}\right) \\
\leq & -\frac{\bar{\alpha}_{1}}{p_{1}} x_{1}^{2}(t)+\frac{2 T \bar{\alpha}_{1}^{2} \bar{\eta}^{2}}{p_{1}^{2}} \sup _{m \in[t-4 T, t]}\left|x_{1}(m)\right|^{2} \\
& +\left|x_{1}(t)\right|\left[\frac{\bar{\alpha}_{1} \bar{\eta}}{p_{1}} \int_{t-2 T}^{t}\left|\xi_{2}(s)\right| \mathrm{d} s+\frac{2 T \bar{\alpha}_{1}^{2} \bar{\eta}}{p_{1}^{2}} \bar{\delta}_{1}\right. \\
& \left.+\left|\xi_{2}(t)\right|+\frac{\bar{\alpha}_{1}}{p_{1}} \bar{\delta}_{1}\right]
\end{aligned}
$$

for all $t \geq T_{e}(|\xi(0)|)+2 T$. Setting $\xi_{\star}^{\sharp}=\xi_{\star}(1+\varepsilon)$, it follows from (39) that, for all $t \geq T_{e}(|\xi(0)|)+2 T$,

$$
\begin{aligned}
\dot{v}(t) \leq & -\frac{\bar{\alpha}_{1}}{p_{1}} x_{1}^{2}(t)+\frac{2 T \bar{\alpha}_{1}^{2} \bar{\eta}^{2}}{p_{1}^{2}} \sup _{m \in[t-4 T, t]}\left|x_{1}(m)\right|^{2} \\
& +\left\{\left|x_{1}(t)\right|\right\}\left\{\frac{2 T \bar{\alpha}_{1} \bar{\eta}}{p_{1}}\left(\xi_{\star}^{\sharp}+\mu_{0}\left(|x(0)|+|\delta|_{\infty}, t\right)\right)\right. \\
& \left.+\frac{2 T \bar{\alpha}_{1}^{2} \bar{\eta} \bar{\delta}_{1}}{p_{1}^{2}}+\xi_{\star}^{\sharp}+\mu_{0}\left(|x(0)|+|\delta|_{\infty}, t\right)+\frac{\bar{\alpha}_{1} \bar{\delta}_{1}}{p_{1}}\right\} \\
\leq & -\frac{2\left(1-\omega_{0}\right) \bar{\alpha}_{1}}{p_{1}} v\left(x_{1}(t)\right) \\
& +\frac{4 T \bar{\alpha}_{1}^{2} \bar{\eta}^{2}}{p_{1}^{2}} \sup _{m \in[t-4 T, t]} v\left(x_{1}(m)\right) \\
& +\frac{p_{1}}{4 \omega_{0} \bar{\alpha}_{1}}\left[\frac{2 T \bar{\alpha}_{1} \bar{\eta}}{p_{1}}\left(\xi_{\star}^{\sharp}+\mu_{0}\left(|x(0)|+|\delta|_{\infty}, t\right)\right)\right. \\
& +\frac{\left.2 T \bar{\alpha}_{1}^{2} \bar{\eta} \frac{\delta_{1}}{p_{1}^{2}}+\xi_{\star}^{\sharp}+\mu_{0}\left(|x(0)|+|\delta|_{\infty}, t\right)+\frac{\bar{\alpha}_{1}}{p_{1}} \bar{\delta}_{1}\right]^{2}}{}
\end{aligned}
$$

where the last inequality applied Young's inequality $a b \leq$ $\frac{\bar{\alpha}_{1} \omega_{0}}{p_{1}} a^{2}+\frac{p_{1}}{4 \bar{\alpha}_{1} \omega_{0}} b^{2}$ to the terms in curly braces.

Third step. By $(12 \mathrm{e})$, we can assume that $\omega_{0} \in(0,1)$ is small enough so that

$$
\frac{\left(1-\omega_{0}\right) \bar{\alpha}_{1}}{p_{1}}>\frac{2 T \bar{\alpha}_{1}^{2} \bar{\eta}^{2}}{p_{1}^{2}}
$$

From Lemma A.1 below and the second inequality in (51), there is a $\mu_{1} \in \mathscr{K} \mathscr{L}$ and a class $\mathscr{M}$ function $T_{e}^{\sharp}:[0,+\infty) \rightarrow$ $[0,+\infty)$ such that for all $t \geq T_{e}^{\sharp}(|\xi(0)|)$, we have

$$
\begin{aligned}
& v\left(x_{1}(t)\right) \leq\left\{\frac { p _ { 1 } ^ { 3 } ( 1 + \omega _ { 0 } ) } { 8 \overline { \alpha } _ { 1 } ^ { 2 } \omega _ { 0 } ( p _ { 1 } ( 1 - \omega _ { 0 } ) - 2 T \overline { \alpha } _ { 1 } \overline { \eta } ^ { 2 } ) } \left[\frac{2 T \bar{\alpha}_{1} \bar{\eta} \xi_{\star}^{\sharp}}{p_{1}}\right.\right. \\
& \left.\left.+\frac{2 T \bar{\alpha}_{1}^{2} \bar{\eta} \bar{\delta}_{1}}{p_{1}^{2}}+\xi_{\star}^{\sharp}+\frac{\bar{\alpha}_{1} \bar{\delta}_{1}}{p_{1}}\right]^{2}\right\}+\mu_{1}\left(|x(0)|+|\delta|_{\infty}, t\right),
\end{aligned}
$$

by the argument that led to (39). Consequently, with the choice $\mu_{2}=\sqrt{2 \mu_{1}}$, we get

$$
\left|x_{1}(t)\right| \leq \gamma_{a}+\mu_{2}\left(|x(0)|+|\delta|_{\infty}, t\right)
$$

where $\gamma_{a}=\sqrt{2 B_{a}}$ and $B_{a}$ is the quantity in curly braces in (52), by the subadditivity of the square root. We next find analogous bounds for $x_{2}$ and $x_{3}$.

From (39) and the fact that $\xi_{2}=x_{2}-\alpha$, and also using (16), we deduce that

$$
\begin{aligned}
& \mid x_{2}(t)+\frac{k^{2}}{\left(1-e^{-k T}\right)^{2}} \int_{t-T}^{t} \int_{m-T}^{m} e^{k(\ell-t)} \frac{\bar{\alpha}_{1}}{p_{1}} \operatorname{sat}_{p_{1}}\left(\eta(\ell) x_{1}(\ell)\right. \\
& \left.+\delta_{1}(\ell)\right) \mathrm{d} \ell \mathrm{d} m \mid \leq \xi_{\star}^{\sharp}+\mu_{0}\left(|x(0)|+|\delta|_{\infty}, t\right)
\end{aligned}
$$

and so also

$$
\begin{aligned}
& \left|x_{2}(t)\right| \leq \xi_{\star}^{\sharp}+\mu_{0}\left(|x(0)|+|\delta|_{\infty}, t\right) \\
& +\frac{k^{2}}{\left(1-e^{-k T}\right)^{2}} \frac{\bar{\alpha}_{1}}{p_{1}} \int_{t-T}^{t} \int_{m-T}^{m} e^{k(\ell-t)}\left[\bar{\eta} \mid x_{1}(\ell)+\overline{\delta_{1}}\right] \mathrm{d} \ell \mathrm{d} m
\end{aligned}
$$

for all $t \geq T_{d}^{\sharp}(|\xi(0)|)$. From this inequality and (53), we deduce that for all $t \geq T_{e}^{\sharp}(|\xi(0)|)+2 T$, we have

$$
\left|x_{2}(t)\right| \leq \xi_{\star}^{\sharp}+\mu_{0}^{\sharp}\left(|x(0)|+|\delta|_{\infty}, t\right)+\frac{\bar{\alpha}_{1}}{p_{1}}\left[\bar{\eta} \gamma_{a}+\overline{\delta_{1}}\right]
$$


where $\mu_{0}^{\sharp}=\mu_{0}+\left(\bar{\alpha}_{1} / p_{1}\right) \bar{\eta} \mu_{2} \in \mathscr{K} \mathscr{L}$. Moreover, since $x_{3}(t)=\dot{\alpha}(t)+\beta(t)+\xi_{3}(t)$, we have

$$
\begin{aligned}
\left|x_{3}(t)\right| \leq & \frac{k^{3}}{\left(1-e^{-k T}\right)^{2}} \int_{t-T}^{t} \int_{m-T}^{m} e^{k(\ell-t)}|\phi(\ell)| \mathrm{d} \ell \mathrm{d} m \\
& +\frac{k^{2}}{\left(1-e^{-k T}\right)^{2}} \int_{t-T}^{t} e^{k(\ell-t)}|\phi(\ell)| \mathrm{d} \ell \\
& +\frac{k^{2}}{\left(1-e^{-k T}\right)^{2}} \int_{t-2 T}^{t-T} e^{k(\ell-t)}|\phi(\ell)| \mathrm{d} \ell \\
& +\frac{k}{1-e^{-k T}} \int_{t-T}^{t} e^{k(\ell-t)}|\omega(\ell)| \mathrm{d} \ell+\left|\xi_{3}(t)\right|,
\end{aligned}
$$

by (16). Next observe that our choices of $y_{1}$ and $y_{2}$ from (2) give

$$
\begin{aligned}
& |\phi(t)| \leq \frac{\bar{\alpha}_{1}}{p_{1}}\left|\eta(t) x_{1}(t)+\delta_{1}(t)\right| \\
& \text { and }|\omega(t)| \leq \frac{\bar{\beta}_{1}}{p_{2}} \mid x_{2}(t)+\delta_{2}(t) \\
& +\frac{k^{2}}{\left(1-e^{-k T}\right)^{2}} \int_{t-T}^{t} \int_{m-T}^{m} e^{k(\ell-t)} \phi(\ell) \mathrm{d} \ell \mathrm{d} m \mid
\end{aligned}
$$

for all $t \geq 2 T$. It follows from (53) and (56) that we can find a function $\mu_{3} \in \mathscr{K} \mathscr{L}$ such that

$$
\begin{aligned}
& |\phi(t)| \leq \phi_{\star}+\mu_{3}\left(|x(0)|+|\delta|_{\infty}, t\right), \\
& \text { where } \phi_{\star}=\frac{\bar{\alpha}_{1}}{p_{1}}\left(\bar{\eta} \gamma_{a}+\bar{\delta}_{1}\right)
\end{aligned}
$$

and $|\omega(t)| \leq \frac{\bar{\beta}_{1}}{p_{2}}\left[\xi_{\star}^{\sharp}+\frac{\bar{\alpha}_{1}}{p_{1}}\left[\bar{\eta} \gamma_{a}+\overline{\delta_{1}}\right]\right]$

$$
\begin{aligned}
& +\frac{\bar{\beta}_{1}}{p_{2}}\left(\mathfrak{s} \int_{t-T}^{t} \int_{m-T}^{m} e^{k(\ell-t)} \mathrm{d} \ell \mathrm{d} m \phi_{\star}+\bar{\delta}_{2}\right. \\
& \left.+\mu_{3}\left(|x(0)|+|\delta|_{\infty}, t\right)\right) \leq \omega_{\star}+\frac{\bar{\beta}_{1}}{p_{2}} \mu_{3}\left(|x(0)|+|\delta|_{\infty}, t\right),
\end{aligned}
$$

by enlarging $T_{e}^{\sharp}$ as needed without relabeling, where $\mathfrak{s}=$ $k^{2} /\left(1-e^{-k T}\right)^{2}$ and where

$$
\omega_{\star}=\frac{\bar{\beta}_{1}}{p_{2}}\left[\xi_{\star}^{\sharp}+\frac{\bar{\alpha}_{1}}{p_{1}}\left(\bar{\eta} \gamma_{a}+\overline{\delta_{1}}\right)+\phi_{\star}+\bar{\delta}_{2}\right] .
$$

Since (16) also gives $|\dot{\alpha}(t)| \leq \frac{2 k|\phi|_{[t-2 T, t]}}{1-e^{-k T}}$ for all $t \geq 2 T$, we deduce from the formula $x_{3}(t)=\dot{\alpha}(t)+\beta(t)+\xi_{3}(t)$ and our bounds on $\phi$ and $\omega$ from (59) and (60) that we can find a class $\mathscr{M}$ function $T_{f}:[0,+\infty) \rightarrow[0,+\infty)$ and a function $\mu_{4} \in \mathscr{K} \mathscr{L}$ such that

$$
\left|x_{3}(t)\right| \leq \frac{2 k}{1-e^{-k T}} \phi_{\star}+\omega_{\star}+\mu_{4}\left(|x(0)|+|\delta|_{\infty}, t\right)
$$

for all $t \geq T_{f}(|\xi(0)|)$, using the formula for $\beta$ from (16).

Fourth step. By combining the upper bounds (53), (56), and (62) for the $\left|x_{i}(t)\right|$ 's and recalling the formulas for the components of $\xi$, we can construct functions $\beta_{0} \in \mathscr{K} \mathscr{L}$ and $\gamma_{0} \in \mathscr{K}_{\infty}$ and a class $\mathscr{M}$ function $T_{g}:[0,+\infty) \rightarrow[0,+\infty)$ such that

$$
|x(t)| \leq \beta_{0}(|x(0)|, t)+\gamma\left(|\delta|_{\infty}\right)
$$

for all $t \geq T_{g}(|x(0)|)$, where the construction of $T_{g}$ used the fact that our formulas (17) imply that there is a class $\mathscr{M}$ function $\Theta:[0,+\infty) \rightarrow[0,+\infty)$ such that $|\xi(0)| \leq \Theta(|x(0)|)$ holds for all initial states $\xi(0)$ and $x(0)$, so we can choose $T_{g}(s)=T_{f}(\Theta(s))$. Also, the linear growth of the $x$ dynamics provides a constant $\bar{L}>0$ such that

$$
\begin{aligned}
& |x(t)| \leq \bar{L} e^{\bar{L} T_{g}(|x(0)|)-t}|x(0)|+\bar{L}|\delta|_{\infty}^{4} \\
& \text { for all } t \in\left[0, T_{g}(|x(0)|)\right] .
\end{aligned}
$$

In fact, (9)-(11) provide a constant $\bar{c}>0$ such that $|\dot{z}(t)| \leq$ $\bar{c}\left(|z|_{[t-T, t]}+|x(t)|+|\delta|_{\infty}\right)$ and therefore also

$$
|z|_{[t-T, t]} \leq \bar{c} \int_{0}^{t}\left(|z|_{[\ell-T, \ell]}+|x(\ell)|\right) \mathrm{d} \ell+\bar{c} t|\delta|_{\infty},
$$

for all $t \geq 0$ (since we assumed that the initial functions for the $z$ dynamics are 0 ). Therefore, we can apply Gronwall's inequality (e.g., from [7, Lemma A.1, p.651]) to the function $\mathscr{F}(t)=|z|_{[t-T, t]}$ to find a constant $\bar{c}^{\sharp}>0$ such that

$$
|z(t)| \leq \bar{c}^{\sharp} e^{\bar{c} T_{g}(|x(0)|)}\left(|x|_{[0, t]}+T_{g}(|x(0)|)|\delta|_{\infty}\right)
$$

for all $t \in\left[0, T_{g}(|x(0)|)\right]$, which we can use to find positive constants $\tilde{c}>0$ and $\bar{c}_{*}$ such that the right side of (1) is bounded by $\tilde{c}\left(e^{\bar{c}_{*} T_{g}(|x(0)|)}|x|_{[0, t]}+|\delta|_{\infty}^{2}\right)$ for all $t \in$ $\left[0, T_{g}(|x(0)|)\right]$ (by applying the triangle inequality to upper bound the term in (66) containing the $|\delta|_{\infty}$ and using the class $\mathscr{M}$ structure of $T_{g}$ ), and then we can apply Gronwall's inequality to the $x$ system (as we did for the $z$ system) to get the required constant $\bar{L}>0$. The final input-to-state stability estimate now follows by adding the bounds for (63)-(64) for $|x(t)|$ to find a bound that holds for all $t \geq 0$.

\section{Application to Visual LANDing of AircRAFT}

To illustrate our results, we consider the lateral dynamics of an Airbus airliner in a glide phase which must align with a runway using a body fixed monocular camera [1]. This problem is a challenge of strong relevance in cases where the runway is unequipped or in the case of GPS loss. More precisely, the position, size and heading of the runway are unknown, so that the relative position $\left(\Delta_{X}, \Delta_{Y}\right)$ and heading $\Delta_{\psi}$ of the aircraft with respect to it are unmeasured. See Figure 1, and see [8] for valuable research on vision based aircraft control, which does not provide the input-to-state stability to uncertainty that we provide in this work.

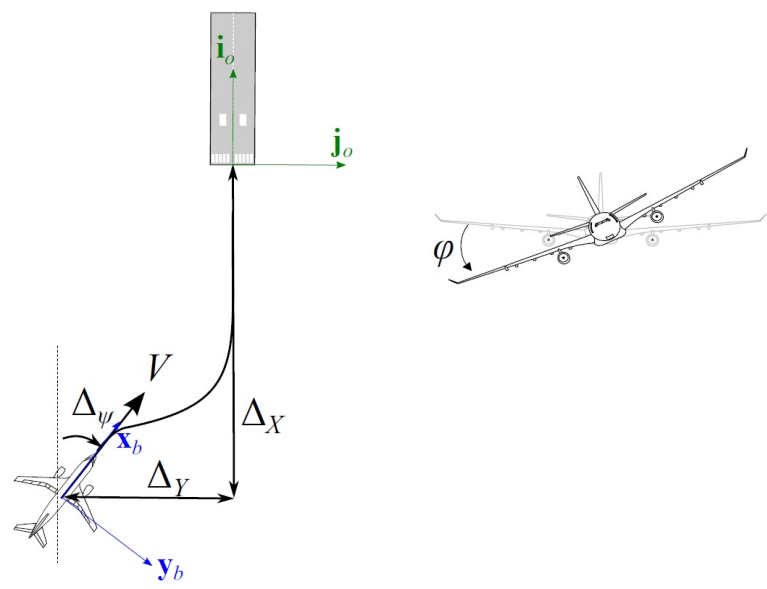

Fig. 1. Notation used in the alignment part of the glide phase

As noted in [11], this research was motivated by this simplified lateral guidance model provided by Airbus:

$$
\left\{\begin{aligned}
\dot{\Delta}_{Y} & =V \operatorname{sat}_{L_{\psi}}\left(\Delta_{\psi}\right) \\
\dot{\Delta}_{\psi} & =\frac{g}{V} \operatorname{sat}_{L_{\varphi}}(\varphi) \\
\dot{\varphi} & =\operatorname{sat}_{L_{u}}\left(u_{\mathrm{lat}}\right)
\end{aligned}\right.
$$


where $u_{\text {lat }}$ is the input, $V=72 \mathrm{~m}^{-1} \mathrm{~s}^{-1}$ is constant all along the final approach, $g=9.81 \mathrm{~m}^{-\mathrm{s}^{-2}}$, and $\varphi$ (resp., $u_{\text {lat }}$ ) is the aircraft roll angle (resp., the guidance/outer loop control action). Then (67) can be transformed into the system (1) by applying the change of coordinates $x_{1}=\Delta_{Y}, x_{2}=V \Delta_{\psi}$, $x_{3}=g \varphi$ while changing the saturation limits accordingly. In [11], the time varying parameter $\eta(t)$ is chosen to satisfy $\eta(t) \in[0.3,1]$, and in our simulations to follow, we scale it so that the new $\eta$ is bounded below by 1 (as explained in Section II above). Note that $\eta$ is minimum when the aircraft is close to the runway and starts its flare.

The saturation limits are

$$
L_{1}=25 m \cdot s^{-1}, L_{2}=7 m \cdot s^{-2}, \text { and } L_{3}=6 m \cdot s^{-3}
$$

For comparison purposes, the controller studied in this paper is called 'controller 1' in our simulations, and we compare its performance with the control from [11] which we refer to as 'controller 2'. We use the folloing parameters the satisfy the assumptions of Theorem 1 when $\bar{\delta}_{1} \leq 0.9$ and $\bar{\delta}_{2} \leq 0.3$ :

$$
\begin{aligned}
& k=0.1, T=2, L_{4}=1, \bar{\alpha}=2, \\
& p_{1}=10, \bar{\beta}=0.2, \text { and } p_{2}=2.5 .
\end{aligned}
$$

It is assumed that the runway suddenly appears in the image at instant $t=5 \mathrm{~s}$ and will remain in the camera field of view throughout the descent. As such, the $x_{i}$ 's are reinitialized at time $t=5 \mathrm{~s}$. Doing so, our controller can be fairly compared to any another one (possibly reacting from $t=0)$. Indeed, according to (4), controller 1 is 0 for $t \in(0,4)$ seconds. Figure 2 shows that controller 1 succeeds to lower a lateral deviation of $30 \mathrm{~m}$ for several initial heading deviations $\Delta_{\psi} \in\{-5,0,5\}$ deg. Figure 3 shows the responses when the outputs are noisy.

On the other hand, our figures also show the performance of the 'controller 2' from [11]. We made these choices of the parameters in 'controller 2' in our simulations:

$$
\begin{aligned}
& k_{1}=0.3, k_{2}=0.59, k_{3}=0.8, \tau=2.5, \\
& L_{4}=1, \text { and } \bar{v}=1.4, \text { and } \phi(x)=0.15 \tanh (0.4 x) .
\end{aligned}
$$

Using these parameters, the assumptions of [11] are satisfied when $\bar{\delta}_{1}=\bar{\delta}_{2}=0$. Moreover, looking at Figures 2 and 3 below, we observe the settling times are almost similar when one uses controllers 1 and 2. However, the assumptions of [11] seem to be far more conservative when the outputs are noisy. Indeed, the assumptions of [11] would not be satisfied with $\bar{\delta}_{1}=0.9$ and $\bar{\delta}_{2}=0.3$, and in order to satisfy them it would be necessary to multiply these bounds by $4.10^{-3}$.

\section{CONCLUSIONS}

We used a recent backstepping approach to construct a useful new class of bounded controls for a chain of saturated integrators that arises in the visual landing of aircraft. This overcame the challenge of having imprecise output measurements by proving an input-to-state stability estimate. In future work, we plan to generalize this work to allow sampling (as described in [18], [19]) or delays in the outputs, which would be useful for modeling the effects of image processing.

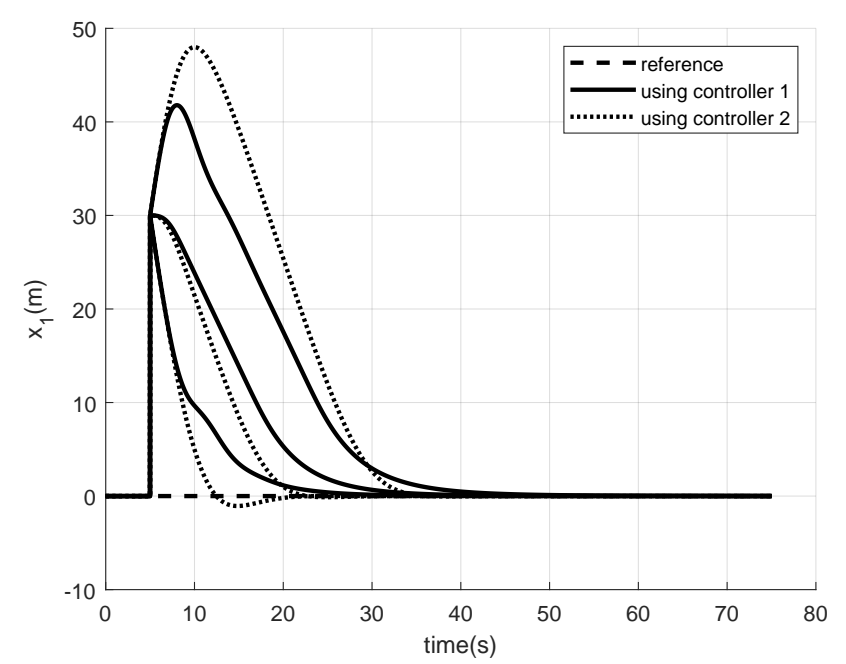

Fig. 2. Evolution of $x_{1}(t)$ when $\bar{\delta}_{1}=0, \bar{\delta}_{2}=0$.

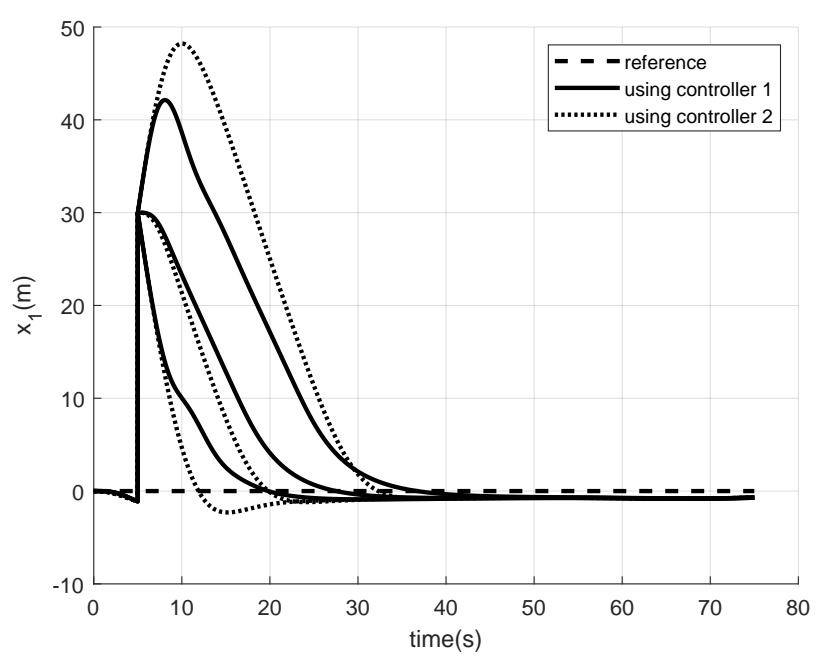

Fig. 3. Evolution of $x_{1}(t)$ when $\bar{\delta}_{1}=0.9, \bar{\delta}_{2}=0.3$.

\section{APPENDIX 1: PROOF OF BOUNDS (15)}

We use the formulas (16) to prove the bounds on the derivatives of $\alpha$ and $\beta$ in (15); the bounds on $\alpha$ and $\beta$ in (15) follow from the definition of the saturation and (16). We use the common notation $\mathfrak{s}=k^{2} /\left(1-e^{-k T}\right)^{2}$ and the equalities and inequalities to follow should be understood to hold for all $t \geq 2 T$. We have

$$
\begin{aligned}
& \dot{\alpha}(t)=-k \alpha(t) \\
& +\mathfrak{s}\left[\int_{t-T}^{t} e^{k(\ell-t)} \phi(\ell) \mathrm{d} \ell-\int_{t-2 T}^{t-T} e^{k(\ell-t)} \phi(\ell) \mathrm{d} \ell\right],
\end{aligned}
$$

and therefore also

$$
\begin{aligned}
|\dot{\alpha}|_{\infty} & \leq k \bar{\alpha}_{1}+\mathfrak{s} \bar{\alpha}_{1}\left[\frac{1}{k}\left(1-e^{-k T}\right)+\frac{1}{k}\left(e^{-k T}-e^{-2 k T}\right)\right] \\
& =k \bar{\alpha}_{1}+\mathfrak{s} \bar{\alpha}_{1} \frac{1}{k}\left(1-e^{-k T}\right)\left(1+e^{-k T}\right)=\bar{\alpha}_{2},
\end{aligned}
$$

by our choice of $\bar{\alpha}_{2}$ in (13). Also, since

$$
\begin{aligned}
& \ddot{\alpha}(t)=-k \dot{\alpha}(t)+\mathfrak{s}(-k)\left[\int_{t-T}^{t} e^{k(\ell-t)} \phi(\ell) \mathrm{d} \ell\right. \\
& \left.-\int_{t-2 T}^{t-T} e^{k(\ell-t)} \phi(\ell) \mathrm{d} \ell\right] \\
& +\mathfrak{s}\left[\phi(t)-2 e^{-k T} \phi(t-T)+e^{-2 k T} \phi(t-2 T)\right],
\end{aligned}
$$


our formula for $\bar{\alpha}_{3}$ from (13) gives

$$
\begin{aligned}
& |\ddot{\alpha}|_{\infty} \leq \frac{2 k^{2} \bar{\alpha}_{1}}{1-e^{-k T}}+\left[\mathfrak{s}\left(1-e^{-k T}\right)+\mathfrak{s}\left(e^{-k T}-e^{-2 k T}\right)\right] \bar{\alpha}_{1} \\
& +\mathfrak{s}\left(1+2 e^{-k T}+e^{-2 k T}\right) \bar{\alpha}_{1} \leq 4 \bar{\alpha}_{1} \mathfrak{s}=\bar{\alpha}_{3} .
\end{aligned}
$$

Finally, since

$$
\dot{\beta}(t)=-k \beta(t)+\frac{k}{1-e^{-k T}}\left(\omega(t)-\omega(t-T) e^{-k T}\right),
$$

we have

$$
|\dot{\beta}|_{\infty} \leq k \bar{\beta}_{1}+\frac{k}{1-e^{-k T}}\left(1+e^{-k T}\right) \bar{\beta}_{1}=\bar{\beta}_{2}
$$

by our choice of $\bar{\beta}_{2}$ in (13). which completes our proof of the bounds (15).

\section{APPENDIX 2: TECHNICAL RESULT}

In this appendix, we provide the variant of the version of Halanay's inequality from [4, Section 4.1.2] that we used in the proof of our theorem. This variant agrees with $[4$, Lemma 4.2] in the special case where $\Delta_{1}=0$.

Lemma A.1: Consider a continuous function $v:[-h,+\infty) \rightarrow[0,+\infty)$. Assume that there are constants $\Delta_{1} \geq 0, \mathfrak{c}_{1}$, and $\mathfrak{c}_{2}$ satisfying $\mathfrak{c}_{1}>\mathfrak{c}_{2}>0$ such that the inequality

$$
\dot{v}(t) \leq-\mathfrak{c}_{1} v(t)+\mathfrak{c}_{2} \sup _{m \in[t-h, t]} v(m)+\Delta_{1}
$$

is satisfied for all $t \geq 0$. Let $c_{s}>0$ be the unique positive value such that $c_{s}=\mathfrak{c}_{1}-\mathfrak{c}_{2} e^{c_{s} h}$, and let $l_{v}>0$ be a constant such that $l_{v} e^{-c_{s} t}>v(t)-\Delta_{1} /\left(\mathfrak{c}_{1}-\mathfrak{c}_{2}\right)$ for all $t \in[-h, 0]$. Then

$$
v(t) \leq l_{v} e^{-c_{s} t}+\frac{\Delta_{1}}{\mathfrak{c}_{1}-\mathfrak{c}_{2}}
$$

holds for all $t \geq 0$.

Proof: First, observe that $\frac{\Delta_{1}}{\mathfrak{c}_{1}-\mathfrak{c}_{2}}$ is well-defined because $\mathfrak{c}_{1}>\mathfrak{c}_{2}$. Let $\tilde{v}(t)=v(t)-\frac{\Delta_{1}}{\mathfrak{c}_{1}-\mathfrak{c}_{2}}$. Then

$$
\dot{\tilde{v}}(t) \leq-\mathfrak{c}_{1} \tilde{v}(t)+\mathfrak{c}_{2} \sup _{m \in[t-h, t]} \tilde{v}(m)
$$

for all $t \geq 0$. Since $\mathfrak{c}_{1}>\mathfrak{c}_{2}$, the required constant $c_{s}$ exists. Also, the function $p(t)=e^{-c_{s} t}$ satisfies

$$
\dot{p}(t)=-\mathfrak{c}_{1} p(t)+\mathfrak{c}_{2} \sup _{m \in[t-h, t]} p(m)
$$

for all $t \geq 0$. It now suffices to prove that $l_{v} p(t) \geq \tilde{v}(t)$ for all $t \geq-h$. To this end, suppose that there is a constant $t_{c}>0$ such that $l_{v} p(t)>\tilde{v}(t)$ for all $t \in\left[-h, t_{c}\right)$ and $l_{v} p\left(t_{c}\right)=\tilde{v}\left(t_{c}\right)$, for the sake of obtaining a contradiction. Let $w(t)=\tilde{v}(t)-$ $l_{v} p(t)$. Then, using $l_{v} p\left(t_{c}\right)=\tilde{v}\left(t_{c}\right)$, we deduce from (A.9) and (A.10) that

$$
\dot{w}\left(t_{c}\right) \leq \mathfrak{c}_{2}\left[\sup _{m \in\left[t_{c}-h, t_{c}\right]} \tilde{v}(m)-\sup _{m \in\left[t_{c}-h, t_{c}\right]} l_{\nu} p(m)\right]<0,
$$

where the last inequality in (A.11) follows because if we choose a $t_{*} \in\left[t_{c}-h, t_{c}\right]$ such that $\sup _{m \in\left[t_{c}-h, t_{c}\right]} \tilde{v}(m)=\tilde{v}\left(t_{*}\right)$, then the quantity in squared brackets in (A.11) is $\tilde{v}\left(t_{*}\right)-$ $l_{v} p\left(t_{c}-h\right)$, which is negative if $t_{*}=t_{c}-h$ (by our choice of $\left.t_{c}\right)$ and is also negative if $t_{*} \in\left(t_{c}-h, t_{c}\right]$ because in that case it is bounded above by $l_{v}\left(p\left(t_{*}\right)-p\left(t_{c}-h\right)\right)<0$. From $\dot{w}\left(t_{c}\right)<0$ and $w(t)<0$ when $t \in\left[-h, t_{c}\right)$, we deduce that $w\left(t_{c}\right)<0$, which is a contradiction. Hence, $w(t)<0$ for all $t \geq-h$. Thus $\tilde{v}(t) \leq l_{v} p(t)$ for all $t \geq-h$, which gives the conclusion.

\section{REFERENCES}

[1] L. Burlion and H. de Plinval, "Vision based anti-windup design with application to the landing of an airliner," IFAC-PapersOnLine, vol. 50, no. 1, pp. 10482-10487, 2017.

[2] G. Chesi and R. Middleton, "LMI-based fixed order output feedback synthesis for two-dimensional mixed continuous-discrete-time systems," IEEE Trans. Autom. Control, vol. 63, no. 4, pp. 960-972, 2018.

[3] J. Gomes da Silva Jr., I. Queinnec, A. Seuret, and S. Tarbouriech, "Regional stability analysis of discrete-time dynamic output feedback under aperiodic sampling and input saturation," IEEE Trans. Autom. Control, vol. 61, no. 12, pp. 4176-4182, 2016.

[4] E. Fridman, Introduction to Time-Delay Systems: Analysis and Control. New York, NY: Springer, 2014.

[5] T. Hu and Z. Lin, Control Systems with Actuator Saturation. Boston, MA: Birkhauser, 2001.

[6] D. Karagiannis and A. Astolfi, "A new solution to the problem of range identification in perspective vision systems," IEEE Trans. Autom. Control, vol. 50, no. 12, pp. 2074-2077, 2005.

[7] H. Khalil, Nonlinear Systems, Third Edition. Englewood Cliffs, NJ: Prentice Hall, 2002.

[8] F. Le Bras, T. Hamel, R. Mahony, C. Barat, and J. Thadasack, "Approach maneuvers for autonomous landing using visual servo control," IEEE Trans. Aerospace and Electronic Systems, vol 50, no. 2, pp. 1051-1065, 2014.

[9] Y. Li and Z. Lin, Stability and Performance of Control Systems with Actuator Saturation. Boston, MA: Birkhauser, 2018.

[10] M. Malisoff and F. Mazenc, Constructions of Strict Lyapunov Functions. London, UK: Springer-Verlag, 2009.

[11] F. Mazenc, L. Burlion, and V. Gibert, "Stabilization with imprecise measurements: application to a vision based landing problem," in Proc. American Control Conf. (IEEE), 2018, pp. 2978-2983.

[12] F. Mazenc, L. Burlion, and M. Malisoff, "Stabilization and robustness analysis for a chain of saturating integrators with imprecise measurements," IEEE Control Systems Letters, vol. 3, no. 2, pp. 428-433, 2019.

[13] F. Mazenc and A. Iggidr, "Backstepping with bounded feedbacks," Systems and Control Letters, vol. 51, no. 3-4, pp. 235-245, 2004.

[14] F. Mazenc and M. Malisoff, "New control design for bounded backstepping under input delay," Automatica, vol. 66, pp. 48-55, 2016.

[15] F. Mazenc, M. Malisoff, L. Burlion, and J. Weston, "Bounded backstepping control and robustness analysis for time-varying systems under converging-input-converging-state conditions," European J. Control, vol. 42, pp. 15-24, 2018.

[16] F. Mazenc, S. Mondié, and S.I. Niculescu, "Global asymptotic stabilization for chains of integrators with a delay in the input," IEEE Trans. Autom. Control, vol. 48, no. 1, pp. 57-63, 2003.

[17] F. Mazenc and L. Praly, "Adding an integration and global asymptotic stabilization of feedforward systems," IEEE Trans. Autom. Control, vol. 41, no 11, pp. 1559-1578, 1996.

[18] A. Palmeira, J. Gomes da Silva Jr., S. Tarbouriech, and I. Ghiggi, "Sampled-data control under magnitude and rate saturating actuators," International J. Robust and Nonlinear Control, vol. 26, no. 15, pp. 3232-3252, 2016.

[19] A. Seuret and J. Gomes da Silva Jr., "Taking into account period variations and actuator saturation in sampled-data systems," Systems and Control Letters, vol. 61, no. 12, pp. 1286-1293, 2012.

[20] R. Sepulchre, M. Jankovic, and P. Kokotovic, Constructive Nonlinear Control. Berlin, Germany: Springer-Verlag, 1996.

[21] S. Tarbouriech, G. Garcia, J. Gomes da Silva Jr., and I. Queinnec, Stability and Stabilization of Linear Systems with Saturating Actuators. Berlin, Germany: Springer-Verlag, 2011.

[22] S. Tarbouriech and J. Gomes da Silva Jr., "Synthesis of controllers for continuous-time delay systems with saturating controls via LMIs," IEEE Trans. Autom. Control, vol. 45, no. 1, pp. 105-111, 2000.

[23] Y. Zhu, M. Krstic, H. Su, and C. Xu, "Linear backstepping output feedback control for uncertain linear systems," International J. Adaptive Control and Signal Processing, vol. 30, no. 8-10, pp. 1080-1098, 2016. 